\title{
Neural monitoring is not a substitute to laryngeal examination in thyroid surgery
}

\author{
Hui Sun XX, \\ Division of Thyroid Surgery, China-Japan Union Hospital of Jilin University, Jilin Provincial Key Laboratory of Surgical Translational Medicine, Changchun city, Jilin Province, China
} Hoon Yub Kim XX ${ }^{*}$,

Department of Surgery, Division of Breast and Endocrine Surgery, Minimally Invasive Surgery and Robotic Surgery Center, KUMC Thyroid Center Korea University, Anam Hospital, Seoul, Korea Gianlorenzo Dionigi XX

Division for Endocrine and Minimally Invasive Surgery, Department of Human Pathology in Adulthood and Childhood “G. Barresi,” University Hospital G. Martino, University of Messina, Via C. Valeria 1, Messina, Italy

\section{A R T I C L E I N F O}

\section{Article history:}

Accepted 20 August 2019

Available online $\mathrm{xxx}$

To the Editor:

We read with interest the article by Schneider et al, "Complete and incomplete recurrent laryngeal nerve injury after thyroid and parathyroid surgery: Characterizing paralysis and paresis," published in Surgery. ${ }^{1}$ The article raises significant questions concerning recurrent laryngeal nerve (RLN) management and factors influencing both the choice and adherence to the pre- and postoperative assessment of vocal cord (VC) mobility. ${ }^{1}$

According to the results, the intraoperative neural monitoring (IONM) findings showed a significant difference in vagal amplitude (V2) after resection. V2 was lower than in the control group but higher than in the VC paralysis group. ${ }^{1}$ A hypothetical damage of a fraction of the nerve fibers in VC paresis resulted in a mildly decreased amplitude compared with the control group, but with sufficient conductive capability, contrary to the VC paralysis group. ${ }^{1}$

We would like to offer a note of caution for endocrine surgeons using IONM and confirm that IONM is not a substitute to pre- (L1) and post-

(L2) laryngeal examination.

1. L1 is the reference for bilateral predissectional vagal nerve (V1) and RLN stimulation and interpretation of EMG data. ${ }^{2}$ L2 is the reference for V2 and postdissection RLN stimulation. ${ }^{2}$

2. The intraoperative assessment of RLN function may not be identical with preoperative VC movement and quality of voice. ${ }^{1-3}$
Some patients with preoperative RLN palsy may not necessarily have pathologic voice quality or a deficient EMG signal. ${ }^{1,4}$

3. L1 is essential whereas IONM is still in the development phase to improve the prognostic correlation between neural stimulation and preoperative glottic function. ${ }^{4}$ The complex neuroanatomy, neurophysiology and neuropathology require a highly RLN anatomy knowledge, combined with an electrophysiologic (IONM) and clinical (L1 and L2) interpretation. ${ }^{4}$

4. IONM has more limits, both technologic and interpretative, than the laryngeal examination. ${ }^{1}$ L1. Furthermore, IONM technology is not widely adopted and needs for standardized and well-trained use to avoid pitfalls. Few surgeons have experience with common IONM pitfalls and troubleshooting algorithms. To solve most nerve monitoring pitfalls, the adherence to L1 and L2 is extremely useful.

5. IONM has a relatively low positive predictive value. ${ }^{1}$

6. Definition of an intraoperative loss of EMG signal implicates a normal VC movement at $\mathrm{L} 1 .^{2}$

7. Patients with preexisting VC palsy revealed reduced amplitude of ipsilateral VN and RLN, indicating retained nerve conductivity despite VC weakened. ${ }^{1}$ L1 is therefore indispensable for a reliable risk assessment by IONM, even in patients without voice abnormalities.

8. IONM technologic failure occurs in about $1.5 \%$ of all procedures. ${ }^{3}$

9. Is it more important in the operative notes a statement that demonstrates that RLN was visualized and functionally intact (IONM), or the pre- and postoperative assessment of VC? L1 and

\footnotetext{
* Corresponding author.

E-mail address: hoonyubkim@gmail.com (H.Y. Kim)
} 
L2 are useful documentation to draw and justify any surgical deliberations, strategies and forensic conclusions. ${ }^{2}$

10. Patient shall beforehand be given appropriate information on the consequences of the intervention. The surgeon is obliged to carefully explain the risks of surgery and the surgical strategies to the patient before surgery. In this perspective, L1 is applied. ${ }^{2}$

11. Preoperative VC edema, granuloma, nodule, ulcer, or laceration may be present even with a normally conducting nerve. These would only be detected on L1. ${ }^{1-4}$

12. IONM costs are higher than laryngeal examination. ${ }^{3}$

\section{Funding/Support}

There are no other funding or financial relationship with the surgical industry.

\section{Conflict of interest/Disclosure}

The authors have no conflict of interest to disclose.

\section{References}

1. M Schneider, V Dahm, C Passler, et al. Complete and incomplete recurrent laryngeal nerve injury after thyroid and parathyroid surgery: Characterizing paralysis and paresis. Surgery. 2019;166:369-374.

2. GW Randolph, D Kamani. The importance of preoperative laryngoscopy in patients undergoing thyroidectomy: Voice, vocal cord function, and the preoperative detection of invasive thyroid malignancy. Surgery. 2006;139:357-362.

3. G Dionigi, A Bacuzzi, L Boni, et al. Visualization versus neuromonitoring of recurrent laryngeal nerves during thyroidectomy: What about the costs? World $\mathrm{J}$ Surg. 2012;36:748-754.

4. K Lorenz, M Abuazab, C Sekulla, et al. Results of intraoperative neuromonitoring in thyroid surgery and preoperative vocal cord paralysis. World J Surg. 2014;38:582-591. 